\title{
Coherent excitation of a two-level atom driven by a far-off-resonant classical field: Analytical solutions
}

\author{
Pankaj K. Jha ${ }^{1, *}$ and Yuri V. Rostovtsev ${ }^{1,2}$ \\ ${ }^{1}$ Institute for Quantum Science and Engineering and Department of Physics, Texas A\&M University, College Station, Texas 77843, USA \\ ${ }^{2}$ Department of Physics, University of North Texas, Denton, Texas 76203, USA
}

(Received 19 May 2009; published 15 March 2010)

\begin{abstract}
We present an analytical treatment of coherent excitation of a two-level atom driven by a far-off-resonant classical field. A class of pulse envelope is obtained for which this problem is exactly solvable. The solutions are given in terms of the Heun function, which is a generalization of the hypergeometric function. Degeneracy of the Heun to a hypergeometric equation can give all the exactly solvable pulse shapes of Gauss hypergeometric form from the generalized pulse shape obtained here. We discuss the application of the results obtained to the generation of soft $\mathrm{x}$-ray and ultraviolet radiations.
\end{abstract}

DOI: 10.1103/PhysRevA.81.033827

PACS number(s): 42.50.-p

\section{INTRODUCTION}

The two-level system (TLS) [1-3] is a very rich and useful model that helps illuminate the physics of many problems, ranging from interaction with electromagnetic fields to level crossing [4-6]. For example, interaction of a beam of atoms in a Stern-Gerlach apparatus [7] and Bloch-Siegert shift [8] can be understood using TLS. Recently TLS has been extensively studied as a quantum bit (qubit) for quantum information theory [9]. Two-level atom (TLA) description is valid if the two atomic levels involved are resonant or nearly resonant with the driving field, while all other levels are highly detuned. The TLS can be realized exactly for a spin- $1 / 2$ system and, approximately, for a multilevel system in a magnetic field when all other magnetic sublevels are detuned far off resonance.

When the frequency of the driving field is in resonance with the atomic transition frequency, the Schrödinger equation for the time evolution of state amplitudes is exactly solvable for any time dependence of the field $\Omega(t)$. The transition probability is given as

$$
p=\sin ^{2}(A / 2), \quad A=\int_{-\infty}^{\infty} \Omega(\tilde{t}) d \tilde{t} .
$$

Here $A$ is the area of the pulse envelope. Interestingly, this transition probability vanishes when $A$ is an even-integer multiple of $\pi$ (CPR). For odd-integer multiples of $\pi$ we get complete population inversion (CPI), while half-integer multiples of $\pi$ give equal coherent superposition of the initial and the final states. Several exactly solvable models for the TLS have been proposed in the past [10-24] where solutions to the Schrödinger equation are expressed in terms of known functions like hypergeomteric functions. Several approximate solutions have also been proposed based on perturbation theory and the adiabatic approximation $[25,26]$.

Recently, the generation of shortwavelength radiation has been a focus of research [27,28]. A two-level atomic system under the action of a far-off-resonant strong pulse of laser radiation has been considered and it has been shown that such pulses can excite remarkable coherence on high-frequency

\footnotetext{
*pkjha@physics.tamu.edu
}

far-detuned transitions; and this coherence can be used for efficient generation of soft $\mathrm{x}$-ray and UV (XUV) radiation [28].

To describe excited coherence, we are interested in understanding the mechanism of breaking adiabaticity that leads to excited coherence in the system when the laser pulse has already passed. Thus we are interested in going beyond classical electrodynamics [29]. Indeed, an electric field that causes polarization of dielectrics is given by

$$
P(t, r)=\int_{-\infty}^{t} d t^{\prime} \chi\left(t-t^{\prime}\right) \mathcal{E}\left(t^{\prime}, r\right),
$$

where $\chi(\tau)$ is the dielectric response function. It is important to note that once the field is removed, the polarization adiabatically returns to practically zero. Breaking of adiabaticity is especially difficult when the frequency of the applied field is far from the atomic resonance. Finding exact analytical solutions for such a problem not only will supplement numerical simulations but also will be useful in understanding the underlying physics.

In this paper, using a proper variable transformation, we find a class of pulse $\Omega(t)$ for which the Schrödinger equation for the time evolution of state amplitudes can be transformed into the well-known Heun equation. The solutions are given in terms of the Heun function, which is a generalization of the hypergeometric function. Using the degeneracy of Heun to hypergeometric equation, the Bambini-Berman model [21] can be generalized to this model.

The paper is organized as follows. In Sec. II, we briefly describe our system and obtain the equation of motion for the state amplitudes. In Sec. III, we present the exact solution of the problem in terms of the local Heun solutions $\mathcal{H l}$. It is well established that the Heun equation reduces to the Gauss hypergeometric equation in several ways so we discuss this degeneracy briefly. We also discuss one of the confluent cases of the Heun equation, that is, the confluent Heun equation, and find the exact solutions. In Sec. IV, we give some specific examples of the pulses for which we have found solutions. Pulse shapes are asymmetric in time except the Rosen-Zener pulse. The hyperbolic secant (Rosen-Zener) model and generalized Rosen-Zener (Bambini-Berman) model are included in this class of $\Omega(t)$ as a special case. We also give a new model for a smooth box pulse which takes care 


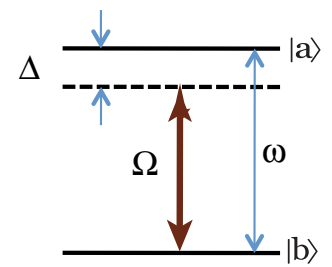

(a)

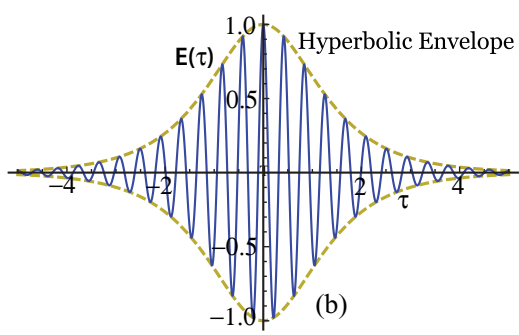

FIG. 1. (Color online) (a) Two-level atomic system: atomic transition frequency $\omega=\omega_{a}-\omega_{b}$, detuning $\Delta=\omega-v$, and Rabi frequency $\Omega(t)=\wp \mathcal{E}(t) / 2 \hbar$. (b) Classical electromagnetic field $E(t)=\operatorname{sech}(\alpha t) \cos (\nu t)$.

of nonanalyticity at the edges by introducing a parameter $\delta$. By modulating this parameter we can modulate the box width. In Sec. V, we discuss the application of the results obtained here to the generation of XUV. We also estimate the level of the XUV field that can be generated by using the excited coherence.

\section{TWO-LEVEL ATOM: EQUATION OF MOTION}

The equation of motion for the probability amplitudes for states $|a\rangle$ and $|b\rangle$ [see Fig. 1(a)] of a TLA interacting with a classical field is given as

$$
\begin{aligned}
\dot{C}_{a} & =i \frac{\wp \mathcal{E}(t)}{\hbar} \cos (v t) e^{i \omega t} C_{b}, \\
\dot{C}_{b} & =i \frac{\wp^{*} \mathcal{E}(t)}{\hbar} \cos (v t) e^{-i \omega t} C_{a},
\end{aligned}
$$

where $\hbar \omega$ is the energy difference between two levels, $\wp$ is the atomic dipole moment, and $E(t)=\mathcal{E}(t) \cos v t$ [see Fig. 1(b)]. In the rotating wave approximation (RWA) we replace $\cos (v t) e^{ \pm i \omega t} \rightarrow e^{ \pm i \Delta} / 2$, where $\Delta=\omega-v$ is detuning from resonance. Introducing $\Omega(t)=\wp \mathcal{E}(t) / 2 \hbar,{ }^{1}$ Eq. (3) reduces to

$$
\begin{gathered}
\dot{C}_{a}=i \Omega(t) e^{i \Delta t} C_{b}, \\
\dot{C}_{b}=i \Omega^{*}(t) e^{-i \Delta t} C_{a},
\end{gathered}
$$

which have an integral of motion $\left|C_{a}\right|^{2}+\left|C_{b}\right|^{2}=1 .^{2}$ There are a variety of ways to approach the problem of solving for $C_{a}(t)$. One method is to define $f(t)=C_{a}(t) / C_{b}(t)$. For the function $f(t)$, Eq. (4) yields the following Riccati equation [28]:

$$
\dot{f}+i \Omega^{*}(t) e^{-i \Delta t} f^{2}-i \Omega(t) e^{i \Delta t}=0 .
$$

Then $\mid C_{a}\left(t|=| f(t) \mid / \sqrt{1+|f(t)|^{2}}\right.$. Alternatively, we can get a second-order linear differential equation for $C_{a}(t)$, from Eq. (4):

$$
\ddot{C}_{a}(t)-\left[i \Delta+\frac{\dot{\Omega}}{\Omega}\right] \dot{C}_{a}(t)+|\Omega|^{2} C_{a}(t)=0 .
$$

\footnotetext{
${ }^{1}$ In this paper we have defined the Rabi frequency as $\Omega(t)=$ $\wp \mathcal{E}(t) / 2 \hbar$ rather than by the usual definition, $\Omega(t)=\wp \mathcal{E}(t) / \hbar$.

${ }^{2}$ Here we consider a TLA with stable levels (or neglect any kinds of decay due to spontaneous emission, collision, etc., on the time scale of the pulse) interacting with a classical external electromagnetic field.
}

The general solution for Eq. (6) has not been found yet, however, there are solutions for several cases in terms of special functions. To find a solution for Eq. (6) we introduce a new variable,

$$
\varphi=\varphi(\tau),
$$

subject to the condition that $\varphi(\tau)$ is a real, positive, and monotonic function of $\tau$ and $\varphi_{0} \leqslant \varphi \leqslant \varphi_{1}$. In terms of the variable $\varphi$ and the dimensionless parameters,

$$
\tau=\alpha t, \quad \beta=\frac{\Delta}{\alpha}, \quad \gamma=\frac{\Omega_{0}}{\alpha},
$$

one may write Eq. (6) for real $\xi(\tau)$ in the form

$$
C_{a}^{\prime \prime}+\left[\frac{\ddot{\varphi} / \dot{\varphi}-i \beta-\dot{\xi} / \xi}{\dot{\varphi}}\right] C_{a}^{\prime}+\frac{\gamma^{2} \xi^{2}}{\dot{\varphi}^{2}} C_{a}=0,
$$

where a prime indicates differentiation with respect to $\varphi$ and $\Omega(\tau)=\gamma \xi(\tau)$. Let us determine the condition under which Eq. (9) has the form

$$
C_{a}^{\prime \prime}(\varphi)+P(\varphi) C_{a}^{\prime}(\varphi)+Q(\varphi) C_{a}(\varphi)=0 .
$$

Using Eqs. (9) and (10) and some trivial algebra, we get

$$
\tau=-\frac{1}{i \beta} \int\left(P+\frac{Q^{\prime}}{2 Q}\right) d \varphi
$$

\section{HEUN EQUATION}

Bambini-Berman studied the case in which Eq. (10) has the form of a Gauss hypergeometric equation which includes the Rosen-Zener model as a special case. Now let us consider when Eq. (10) is in the form of the Heun equation ${ }^{3}[30,31]$ with the independent variable $\varphi$ :

$\frac{d^{2} C_{a}}{d \varphi^{2}}+\left(\frac{u}{\varphi}+\frac{v}{\varphi-1}+\frac{w}{\varphi-c}\right) \frac{d C_{a}}{d \varphi}+\frac{(a b \varphi-q) C_{a}}{\varphi(\varphi-1)(\varphi-c)}=0$,

where $a, b, c, q, u, v, w$ are parameters with $c \neq 0,1 \quad(c>$ $1)$. The parameters are constrained, by the general theory of Fuchsian equations, as

$$
u+v+w=a+b+1 .
$$

From Eqs. (10) and (12) and some algebra, we get

$$
\dot{\varphi}=2 \varphi(1-\varphi) /(\mu+\lambda \varphi) \text {. }
$$

Equivalently, the parameters of the Heun equation, Eq. (12), are given as

$$
\begin{aligned}
& u=\frac{1}{2}-\frac{i \beta \mu}{2}, \quad v=\frac{1}{2}+\frac{i \beta(\lambda+\mu)}{2}, \quad w=\frac{1}{2}, \\
& a=0, \quad b=\frac{1}{2}-\frac{i \beta \lambda}{2} .
\end{aligned}
$$

\footnotetext{
${ }^{3}$ Heun equation: In general for real $\Omega(\tau)$, we get an additional constraint for our asymmetric parameters, $q<0$ and $a b / q<1$ if $q \neq 0$, or $a b>0$ if $q=0$. Confluent Heun equation: $q<0$ and $p / q>-1$ if $q \neq 0$, or $p<0$ if $q=0$.
} 


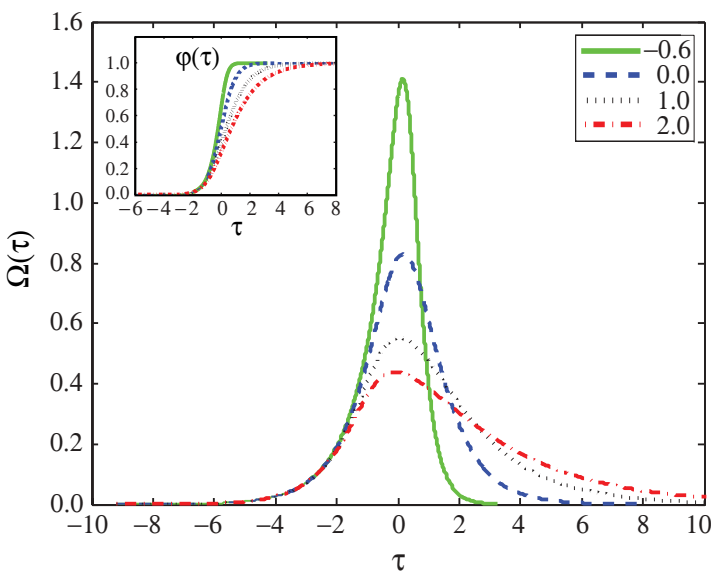

(a)

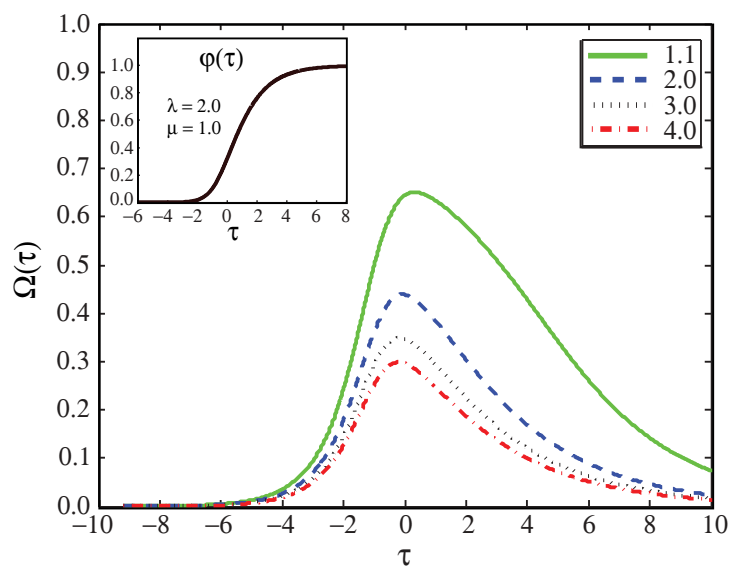

(b)

FIG. 2. (Color online) Pulse shapes given by Eq. (18). (a) Pulse shapes with varying $\lambda$ and $c=2, q=-1$, and $a b=0$. (b) Pulse shapes with varying $c$ and $\lambda=2, q=-1$, and $a b=0$.

For $\varphi(\tau)$ to be a monotonically increasing function of $\tau, \dot{\varphi}$ must be real and positive, that is, $\mu>0$ and $\lambda / \mu>-1$. The time variable $\tau$ as a function of $\varphi$ is obtained by integrating Eq. (14), which gives

$$
2 \tau=\ln \left[\varphi^{\mu} /(1-\varphi)^{\mu+\lambda}\right] .
$$

The general solution for Eq. (12), which has regular singularity at $\varphi=0$, is given in terms of the Heun local solutions, $\mathcal{H l}(\varphi)$, as

$$
\begin{aligned}
C_{a}= & \mathcal{P}_{1} \varphi^{1-u} \mathcal{H} l\{c, q+(1-u)[(c-1) v+a+b-u+1], \\
a- & u+1, b-u+1,2-u, v ; \varphi\} \\
& +\mathcal{P}_{2} \mathcal{H} l[c, q ; a, b, u, v ; \varphi]
\end{aligned}
$$

where the constants $\mathcal{P}_{1}, \mathcal{P}_{2}$ can be found using the initial conditions of the system. In the limit $\tau \rightarrow \infty$, the population left in level $|a\rangle$ can be obtained by substituting $\varphi \rightarrow 1$ in Eq. (17). The form of the pulse can be obtained by equating Eqs. (9) and (12), which gives

$$
\Omega(\tau)=\left[\frac{4 \varphi(1-\varphi)(a b \varphi-q)}{(c-\varphi)}\right]^{1 / 2}\left(\frac{1}{\mu+\lambda \varphi}\right),
$$

where $\varphi(\tau)$ is given by Eq. (16). In Fig. 2 we have plotted the pulse envelopes of the classical field, given by Eq. (18), for which the TLA problem can be exactly solved. They also show the effect of the asymmetric parameters $\lambda$ and $c$, respectively, for $\mu=1$, on the symmetry of the shapes. Pulse shapes showing the effects of other parameters can also be plotted easily from Eq. (18).

There are three kinds of solutions to the Heun equation, Eq. (12): local solutions $\mathcal{H} l$, Heun functions $\mathcal{H} f$, and Heun polynomials $\mathcal{H} p$ [32-34]. The series solution, Eq. (17), is written as [31]

$\mathcal{H} l[c, q ; a, b, u, v ; \varphi]=\sum_{j=0}^{\infty} s_{j} \varphi^{j}=1+\frac{q}{u c} \varphi+\sum_{j=2}^{\infty} s_{j} \varphi^{j}$, where $s_{j}$ obeys the three-term recursion relation,

$$
\begin{gathered}
(j-1+a)(j-1+b) s_{j-1}-\{j[(j-1+u)(1+c)+v c \\
+a+b+1-u-v]+q\} s_{j}+(j+1)(j+u) s_{j+1}=0,
\end{gathered}
$$

with the initial conditions

$$
s_{0}=1, \quad s_{1}=\frac{q}{u c}, \quad \text { and } \quad s_{j}=0, \quad \text { if } \quad j<0 .
$$

The solution, Eq. (19), is valid only within a circle centered at the origin $\varphi=0$ whose radius is the distance from the origin to the nearest singularity $\varphi=1$ or $\varphi=c$. For $c>1$, the radius of convergence is 1 [31]. From Eq. (20), we can say that the Heun function remains the same with the exchange of parameters $a$ and $b$.

\section{A. Degeneracy to hypergeometric models}

It can be easily verified that the Heun equation, Eq. (12), can be reduced to the hypergeometric equation in several ways [31]:

$$
\begin{gathered}
c=1, \quad q=a b, \\
w=0, \quad q=c a b, \\
c=0, \quad q=0 .
\end{gathered}
$$

Let us now consider the simplest case of $c=0, q=0$. Then for $a+b=0$ and $1 / 2-v=-i \beta / 2$, Eq. (12) reduces to the standard form of the Gauss hypergeometric equation:

$$
\frac{d^{2} C_{a}}{d \varphi^{2}}+\left[\frac{r-(1+a+b) \varphi}{\varphi(1-\varphi)}\right] \frac{d C_{a}}{d \varphi}-\frac{a b C_{a}}{\varphi(1-\varphi)}=0,
$$

where $r=1 / 2-i \beta / 2$. The general solution for Eq. (23) is

$$
\begin{aligned}
C_{a}= & \mathcal{P}_{1} \varphi^{1-r} \mathcal{F}[b-r+1, a-r+1 ; 2-r ; \varphi] \\
& +\mathcal{P}_{2} \mathcal{F}[a, b ; r ; \varphi],
\end{aligned}
$$

where the constants, $\mathcal{P}_{1}, \mathcal{P}_{2}$ can be found using the initial conditions of the problem. We write the hypergeometric series $\mathcal{F}_{(2,1)}[a, b ; c ; \varphi]$ as $\mathcal{F}[a, b ; c ; \varphi]$. The population left in state 
$|a\rangle$ is given as

$C_{a f}=\mathcal{P}_{1} \mathcal{F}[b-r+1, a-r+1 ; 2-r ; 1]+\mathcal{P}_{2} \mathcal{F}[a, b ; r ; 1]$.

Subsequently, if $(a+b)=\lambda i \beta$ and $v-1 / 2-(a+b)=$ $\mu i \beta$, we have the generalized Rosen-Zener model as discussed by Bambini and Berman [21]. One can summarize the degeneracy of the Heun to the hypergeometric model as follows:

$$
\begin{gathered}
\mathcal{H} l[1, a b ; a, b, u, v ; \varphi]=\mathcal{F}[a, b ; u ; \varphi], \\
\mathcal{H} l[c, c a b ; a, b, u, a+b-u+1 ; \varphi]=\mathcal{F}[a, b ; u ; \varphi], \\
\mathcal{H} l[0,0 ; a, b, u, v ; \varphi]=\mathcal{F}[a, b ; a+b-v+1 ; \varphi] .
\end{gathered}
$$

\section{B. Confluent Heun equation}

The confluent Heun equation is one of the four confluent forms of Heun's equation, which is obtained by merging the singularity at $\varphi=c$ with that at $\varphi=\infty$. Now we have a regular singularity at $\varphi=0,1$ and an irregular singularity at $\varphi=\infty$. In this paper we consider the following nonsymmetrical form of the confluent Heun equation:

$$
\frac{d^{2} C_{a}}{d \varphi^{2}}+\left(\frac{u}{\varphi}+\frac{v}{\varphi-1}\right) \frac{d C_{a}}{d \varphi}+\frac{p \varphi+q}{\varphi(\varphi-1)} C_{a}=0 .
$$

Similarly to the Heun case, we have the same differential equation for $\dot{\varphi}$, that is, Eq. (14). For the confluent Heun equation, the possible values of the asymmetric parameters are

$$
\begin{gathered}
u=\frac{1}{2}-\frac{i \beta \mu}{2}, \quad v=\frac{i \beta(\lambda+\mu)}{2}, \quad p=-q, \\
u=\frac{1}{2}-\frac{i \beta \mu}{2}, \quad v=\frac{1}{2}+\frac{i \beta(\lambda+\mu)}{2}, \quad p=0
\end{gathered}
$$

The general solution of the confluent Heun equation, Eq. (27), is given as

$$
\begin{aligned}
C_{a}= & \mathcal{P}_{1} \mathcal{H} l^{(c)}[0, u-1, v-1, p, q+(1-u v) / 2, \varphi] \\
& +\mathcal{P}_{2} \varphi^{1-u} \mathcal{H} l^{(c)}[0,1-u, v-1, p, q+(1-u v) / 2, \varphi],
\end{aligned}
$$

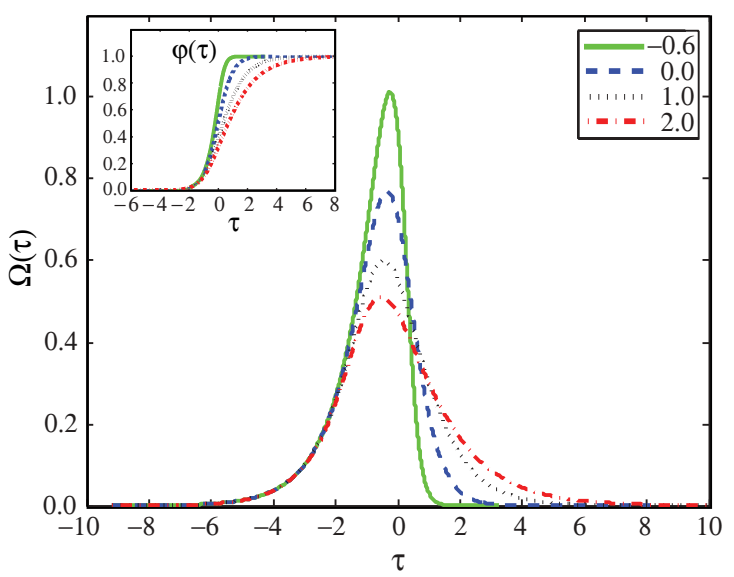

(a) where $\mathcal{P}_{1}, \mathcal{P}_{2}$ can be found using the initial condition of the system. It is worth mentioning here that the general solution to the Gauss hypergeometric differential equation, Eq. (23), can be expressed in terms of the Heun functions $\mathcal{H} l^{(c)}$ as

$$
\begin{aligned}
C_{a}= & \mathcal{P}_{1}(\varphi-1)^{-a} \mathcal{H} l^{(c)}\{0, a-b,-1+r, 0,[(r-2 a) b-r \\
& +r a+1] / 2,1 /(1-\varphi)\}+\mathcal{P}_{2}(\varphi-1)^{-b} \mathcal{H} l^{(c)}\{0, b-a, \\
& -1+r, 0,[(r-2 a) b-r+r a+1] / 2,1 /(1-\varphi)\} .
\end{aligned}
$$

The form of the pulse can be obtained by equating Eqs. (9) and (27), which gives

$$
\Omega(\varphi)=\frac{[4 \varphi(\varphi-1)(p \varphi+q)]^{1 / 2}}{\mu+\lambda \varphi},
$$

where $\varphi(\tau)$ is given by Eq. (16). The constraint of $\lambda$ and $\mu$ is also the same as for the Heun case discussed earlier. Figure 3 shows the pulse shapes for which the TLA can be reduced to the confluent Heun equation. It also qualitatively shows the effect of the asymmetric parameters $\lambda$ and $q$ on the symmetry of the pulse shapes. $\lambda=0$ corresponds to the symmetric pulse.

\section{SOME EXAMPLES}

In this section we consider some specific examples of pulses corresponding to the Heun and confluent Heun equations. Interestingly, we will also find a better approximation for a box pulse by introducing a parameter $\delta$, which takes care of nonanalyticity of the pulse at the edges.

\section{A. $\Omega_{\delta}(t)=\Omega_{0} \operatorname{sech}(\alpha t) / \sqrt{\delta-\tanh (\alpha t)}, \quad \delta>1$}

For this pulse, using the scaling parameters, Eqs. (8) and (6), gives

$$
\begin{aligned}
& \ddot{C}_{a}(\tau)-\left[i \beta+\frac{1}{2}\left(\frac{1-2 \delta \tanh \tau+\tanh ^{2} \tau}{\delta-\tanh \tau}\right)\right] \dot{C}_{a}(\tau) \\
& +\frac{\gamma^{2} \operatorname{sech}^{2} \tau}{\delta-\tanh \tau} C_{a}(\tau)=0
\end{aligned}
$$

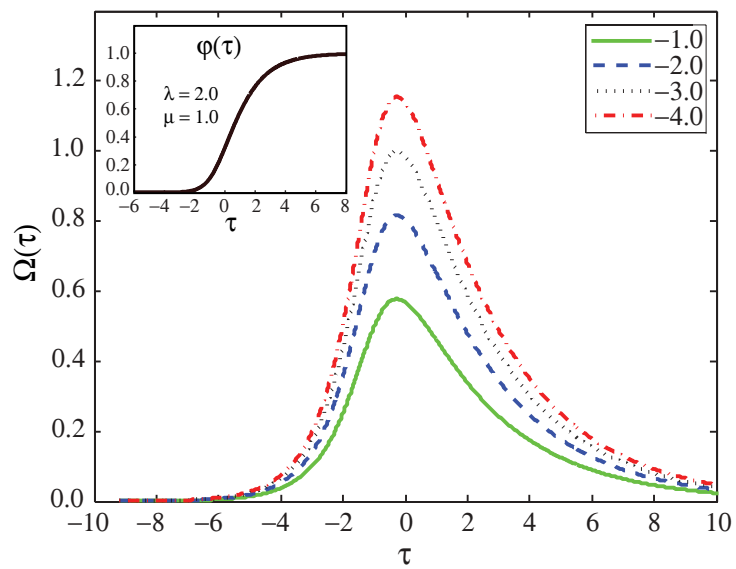

(b)

FIG. 3. (Color online) Pulse shapes given by Eq. (31). (a) Pulse shapes with varying $\lambda$ and $p=-q=1$. (b) Pulse shapes with varying $q$, $\lambda=2$, and $p=0$. 
Let us now define a new variable as

$$
\varphi(\tau)=\frac{1+\tanh \tau}{2} .
$$

In terms of the variable $\varphi$, Eq. (32) reduces to the Heun equation,

$$
C_{a}^{\prime \prime}+\left[\frac{u}{\varphi}+\frac{v}{\varphi-1}+\frac{w}{\varphi-c}\right] C_{a}^{\prime}+\frac{a b \varphi-q}{\varphi(\varphi-1)(\varphi-c)} C_{a}=0
$$

where

$$
\begin{gathered}
u=\frac{1}{2}-\frac{i \beta}{2}, \quad v=\frac{1}{2}+\frac{i \beta}{2}, \quad w=\frac{1}{2}, \\
q=-\frac{\gamma^{2}}{2}, \quad a=0, \quad b=\frac{1}{2} \quad c=\frac{\delta+1}{2} .
\end{gathered}
$$

From Eq. (32) we see that as $\tau \rightarrow-\infty, \varphi \rightarrow 0$, and as $\tau \rightarrow$ $\infty, \varphi \rightarrow 1$. The initial conditions for our system are

$$
C_{a}(\tau \rightarrow-\infty)=0, \quad\left|C_{b}(\tau \rightarrow-\infty)\right|=1 .
$$

The complete solution to Eq. (34), satisfying the initial conditions, Eq. (36), is

$$
\begin{aligned}
C_{a}(\varphi)= & \frac{\gamma \sqrt{2}}{(i-b) \sqrt{c}} \varphi^{1-u} \mathcal{H} l\{c, q+(1-u)[(c-1) v \\
& +a+b-u+1] ; b-u+1, a-u+1,2-u, v, \varphi\} .
\end{aligned}
$$

where $a, b, c, q, u, v, w$ are given by Eq. (35). Let us now consider a case in which $\delta=1$. So the pulse has the form

$$
\Omega_{1}(t)=\Omega_{0} \sqrt{1+\tanh (\alpha t)} .
$$

Now for this pulse, using the scaling parameters, Eq. (8), Eq. (6) gives

$$
\begin{aligned}
& \ddot{C}_{a}(\tau)-\left[i \beta+\frac{1}{2}(1-\tanh \tau)\right] \dot{C}_{a}(\tau)+\gamma^{2}(1+\tanh \tau) C_{a}(\tau) \\
& \quad=0 .
\end{aligned}
$$

In terms of the variable $\varphi$, Eq. (39) reduces to

$$
C_{a}^{\prime \prime}+\left[\frac{u}{\varphi}+\frac{v}{\varphi-1}\right] C_{a}^{\prime}+\frac{q}{\varphi(\varphi-1)^{2}} C_{a}=0,
$$

where

$$
u=\frac{1}{2}-\frac{i \beta}{2}, \quad v=1+\frac{i \beta}{2}, \quad q=\frac{\gamma^{2}}{2} .
$$

The general solution to Eq. (40) is

$$
\begin{aligned}
C(\varphi)_{a}= & \mathcal{P}_{1}(\varphi-1)^{\xi} \mathcal{F}[\xi, \xi-1+u+v ; u ; \varphi] \\
& +\mathcal{P}_{2} \varphi^{1-u}(\varphi-1)^{\xi} \mathcal{F}[\xi+v, \xi+1-u ; 2-u ; \varphi],
\end{aligned}
$$

where

$$
\xi=\frac{1-v}{2}+\sqrt{\left(\frac{1-v}{2}\right)^{2}-q},
$$

and $q, u, v$ are given by Eq. (41). Using the initial conditions, Eq. (36), we get $\mathcal{P}_{1}=0$ and

$$
\mathcal{P}_{2}=\frac{\gamma}{\sqrt{2}(u-1)(-1)^{(\xi+1 / 2)}} .
$$

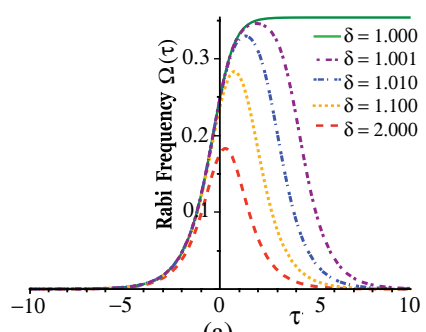

(a)

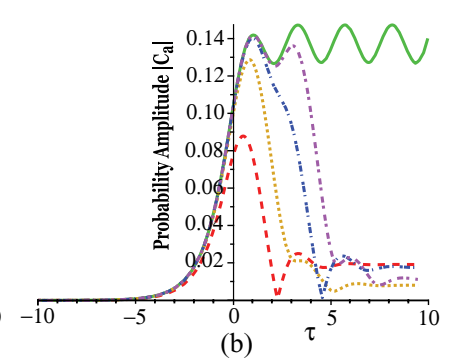

(b)
FIG. 4. (Color online) (a) Pulse shapes for different values of $\delta$. (b) Time dependence of the population in state $|a\rangle$ for a $\Omega_{\delta}(\tau)$ pulse for different values of $\delta>1$. For calculation we take $\alpha=0.08 \omega_{c}$, varying $\delta$.

Figure 4 shows the plot of the population in state $|a\rangle$ corresponding to the pulse $\Omega_{\delta}$ satisfying the initial condition.

\section{B. $\Omega_{+}(t)=\Omega_{0} \operatorname{sech}(\alpha t) \sqrt{1+\tanh (\alpha t)}$}

For this pulse, using the scaling parameters, Eqs. (6) and (8), gives

$$
\begin{aligned}
& \ddot{C}_{a}(\tau)-\left[i \beta+\frac{1}{2}(1-3 \tanh \tau)\right] \dot{C}_{a}(\tau) \\
& \quad+\gamma^{2} \operatorname{sech}^{2} \tau(1+\tanh \tau) C_{a}(\tau)=0 .
\end{aligned}
$$

In terms of the new variable $\varphi$, Eq. (45) reduces to the confluent Heun equation,

$$
C_{a}^{\prime \prime}+\left[\frac{u}{\varphi}+\frac{v}{\varphi-1}\right] C_{a}^{\prime}+\frac{\sigma}{\varphi-1} C_{a}=0,
$$

where

$$
u=-\frac{i \beta}{2}, \quad v=\frac{1}{2}+\frac{i \beta}{2}, \quad \sigma=-2 \gamma^{2} .
$$

The complete solution to Eq. (46) satisfying the initial conditions, Eq. (36), is

$$
\begin{aligned}
C_{a}(\varphi)= & \left(\frac{2 \sqrt{2} \gamma}{2 i-\beta}\right) \varphi^{1+(i \beta / 2)} \mathcal{H} l^{(c)}[0,1+i \beta / 2,-1 / 2+i \beta / 2, \\
& \left.-2 \gamma^{2}, 1 / 2-\beta^{2} / 8-i \beta / 8, \varphi\right] .
\end{aligned}
$$

\section{C. $\Omega_{-}(t)=\Omega_{0} \operatorname{sech}(\alpha t) \sqrt{1-\tanh (\alpha t)}$}

For this pulse, using the scaling transformation, Eq. (8), Eq. (6) gives

$$
\begin{aligned}
& \ddot{C}_{a}(\tau)-\left[i \beta-\frac{1}{2}(1+3 \tanh \tau)\right] \dot{C}_{a}(\tau) \\
& \quad+\gamma^{2} \operatorname{sech}^{2} \tau(1-\tanh \tau) C_{a}(\tau)=0 .
\end{aligned}
$$

In terms of the new variable $\varphi$, Eq. (49) reduces to the confluent Heun equation,

$$
C_{a}^{\prime \prime}+\left[\frac{u}{\varphi}+\frac{v}{\varphi-1}\right] C_{a}^{\prime}+\frac{\eta}{\varphi} C_{a}=0,
$$

where

$$
u=\frac{1}{2}-\frac{i \beta}{2}, \quad v=\frac{i \beta}{2}, \quad \eta=2 \gamma^{2}
$$




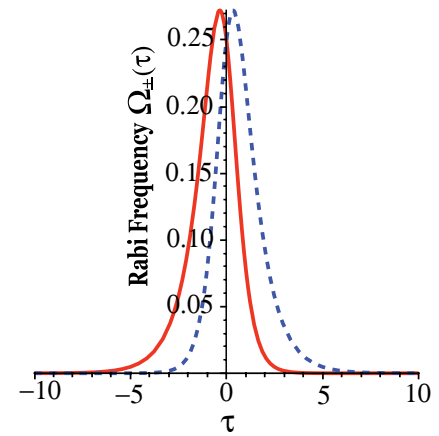

(a)

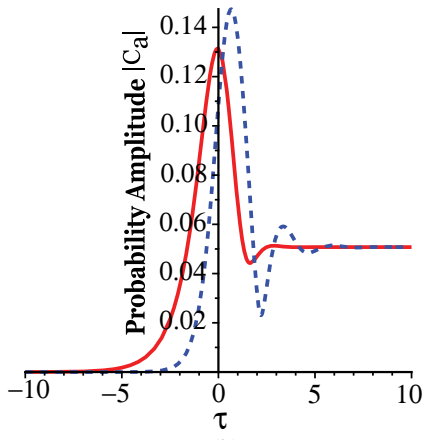

(b)
FIG. 5. (Color online) (a) Pulse shapes for $\Omega_{ \pm}(t)=$ $\Omega_{0} \operatorname{sech} \alpha t(\sqrt{1 \pm \tanh \alpha t})$. (b) Time dependence of the population in state $|a\rangle$ for the pulse shapes in (a). In calculations we take $\Omega_{0}=0.02 \omega_{c}, \alpha=0.08 \omega_{c}$, and $\Delta=0.2 \omega_{c}$.

The complete solution to Eq. (50), satisfying the initial conditions, Eq. (36), is

$$
\begin{aligned}
C_{a}(\varphi)= & \left(\frac{2 \sqrt{2} \gamma}{\beta-i}\right) \varphi^{\frac{1}{2}+\frac{i \beta}{2}} \mathcal{H} l^{(c)}[0,1 / 2+i \beta / 2,-1 \\
& \left.+i \beta / 2,2 \gamma^{2}, 1 / 2-2 \gamma^{2}-\beta^{2} / 8-i \beta / 8, \varphi\right]
\end{aligned}
$$

In Fig. 5 we have plotted the pulse shapes $\Omega_{ \pm}(\tau)$ and the corresponding time evolution of the probability amplitude for state $|a\rangle$.

\section{Smooth box pulse}

One of the simplest and exactly solvable pulse shapes is a box pulse. Indeed it is a nonanalytical pulse but it gives information about the basic oscillatory nature of the solution (probability amplitude). Let us define our pulse as

$$
\Omega(t)=\Omega_{0} \Theta(t) \Theta\left(t_{0}-t\right), \quad t_{0}>0,
$$

where $\Theta(t)$ is a unit step function. The solution for Eq. (6) corresponding to the box pulse is

$$
C_{a}(t)=\frac{i \Omega_{0}}{\sqrt{\Delta^{2} / 4+\Omega_{0}^{2}}} e^{i(\Delta / 2) t} \sin \left(\sqrt{\Delta^{2} / 4+\Omega_{0}^{2}}\right) t, \quad t<t_{0}
$$

The oscillatory nature of the solution $|C(t)|$ is evident from the sine function. Let us consider the pulse shape of the form

$$
\Omega_{\delta}(t)=\frac{\Omega_{0} \operatorname{sech}(\alpha t)}{\sqrt{\delta-\tanh (\alpha t)}}, \quad \delta=2 c-1
$$

where $\mathrm{c}$ is one of the singularities of the Heun equation. Assuming $c>1$ gives $\delta>1$. A pulse shape of the form of Eq. (55) is positive definite and it vanishes at $\tau= \pm \infty$. Let us see what happens when $\delta$ approaches but never reaches 1 . We see from [Figs. 4(a) and 6(a)] that as $\delta$ approaches 1 , the pulse becomes broader and broader, thereby making it a better approximation for a box pulse (taking care of nonanalyticity at the edges). The general solution for a pulse of the form Eq. (55) is given by Eq. (17), where the asymmetric parameters are given by Eq. (35).

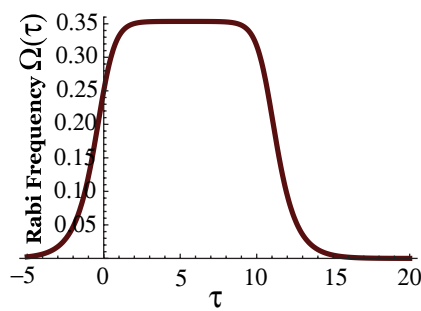

(a)

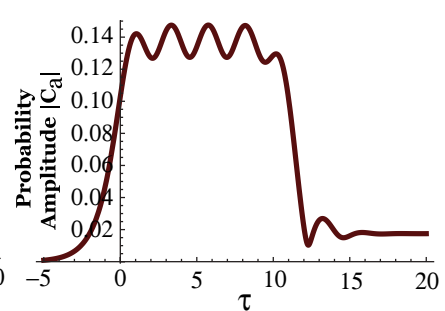

(b)
FIG. 6. (a) Box pulse for $\delta-1=10^{-9}$. (b) Time dependence of the population in state $|a\rangle$ for the box pulse $\Omega_{\delta}(\tau)$. In calculations we take $\Omega_{0}=0.02 \omega_{c}, \alpha=0.08 \omega_{c}$, and $\Delta=0.2 \omega_{c}$.

\section{DISCUSSION}

The obtained results can be applied to the generation of XUV radiation, which is one of the main topics in modern optoelectronics and photonics [35]. Recent progress in ultrashort, for example, attosecond, laser technology allows searchers to obtain ultrastrong fields [36]. Interaction of such strong and broadband fields with a TLA system, even under the action of a far-off-resonance laser radiation is of current interest [27,28,37-39]. Strong, short laser pulses can excite remarkable coherence in high-frequency transitions, and this coherence can be used for surprisingly efficient generation of XUV radiation $[27,28,38,39]$. In the first step we excite the atoms (e.g., from the $1 s$ to the $2 s$ state of $\mathrm{H}$ or $\mathrm{He}^{+}$) via a short pulse of femto- or attosecond radiation, for example, from a conventional Ti:sapphire laser system. The excitation occurs due to the coherent coupling between $1 s$ and $2 p$ and then between $2 p$ and $2 s$. In the second step, we apply another pulse, which scatters off the Raman coherence (prepared in the first step), generating short-wavelength antiStoke radiation as depicted in Fig. 7. The generation of radiation is a coherent process that (contrary to conventional superfluorescence) does not require population inversion (see the Appendix). The higher efficiency of the coherent process has been demonstrated in various spectral regions [40-47].

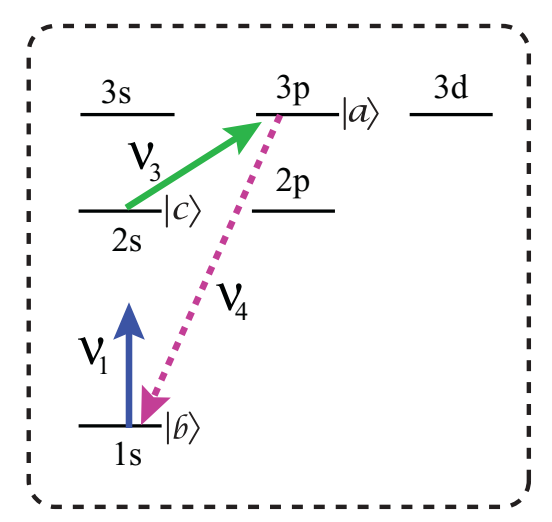

FIG. 7. (Color online) Field configuration and level structure of $\mathrm{H}$ or $\mathrm{He}^{+}$. All population is initially in the ground state $|b\rangle$. First, a strong, short, far-of-resonant pulse with frequency $v_{1}$ is applied to the system to excite coherence between level $|b\rangle=|1 s\rangle$ and level $|c\rangle=|2 s\rangle$, and then a second pulse, with frequency $\nu_{3}$, which is close to the transition between level $2 s$ and level $2 p$, is applied to generate a XUV pulse with a higher frequency $v_{4}$. 


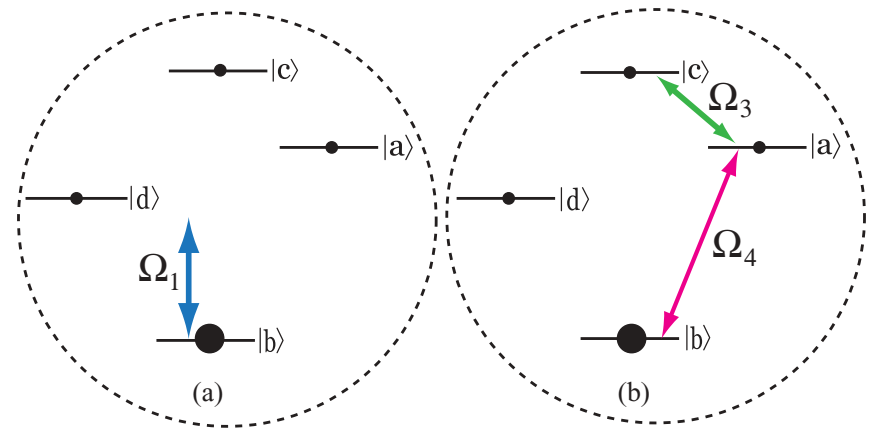

FIG. 8. (Color online) Two-stage generation scheme for x-ray generation. (a) Applying a strong pulse allows one to excite an atomic system by transferring the population to electronic excited states. (b) Coherence is then induced by applying a resonant field.

We have analytically calculated above that the level of excited coherence when a TLA is driven by an ultrashort intense pulse. The coherence is sufficiently large that this can be used for nonlinear generation of XUV radiation, that is, coherence can be of the order of 0.1 [see Figs. 4(b), 5(b), and 6(b)]. It is instructive to estimate the level of XUV field that can be generated by using this coherence. After an ultrastrong and short pulse, we apply a strong resonant and relatively long pulse. The applied probe pulse $\Omega_{3}$ and generated signal $\Omega_{4}$ are coupled to each other via coherence excited in the medium (Rabi frequencies are defined as $\Omega_{3,4}=\wp_{3,4} E_{3,4} / \hbar$ ). Hence, the propagation equation for $\Omega_{4}$ is given by

$$
\frac{\partial \Omega_{4}}{\partial z}=-i \eta_{4} \rho_{a b},
$$

where $\rho_{a b}$ is the appropriate atomic coherence (see Fig. 8) and $\eta_{4}=k_{4} \wp_{4}^{2} N / 2 \hbar$, where $\wp_{4}$ is the dipole moment at the transition between. The corresponding equation for the density matrix coherence $\rho_{a b}$ is

$$
\dot{\rho}_{a b}=-\Gamma_{a b} \rho_{a b}+i \Omega_{4}\left(\rho_{a a}-\rho_{b b}\right)-i \Omega_{3} \rho_{c b},
$$

and for short pulses, $\rho_{a b} \simeq-i \Omega_{3} \tau \rho_{c b}$. Then we can estimate the intensity of the signal field by

$$
\Omega_{4}=\left[\frac{k_{4} L \wp_{a b}^{2} N}{2 \hbar}\right] \rho_{c b} \Omega_{3} \tau,
$$

where $k_{4}$ is the wave number for signal radiation, $L$ is the length of the active medium, $\wp_{a b}$ is the dipole moment at the transition between $a$ and $b$ levels, and $\tau$ is the time duration of the pump laser pulse. Using the parameters $N \simeq$ $10^{16-19} \mathrm{~cm}^{-3}, \wp_{a b} \simeq 1 D, L=100 \mu \mathrm{m}, \rho_{c b}=10^{-1}, \Omega_{3} \tau=$ $1-10^{3}, \tau=1 \mathrm{ps}$, and $\lambda=10 \mathrm{~nm}$, we obtain energy of $\simeq 10$ $\mathrm{nJ}-1 \mu \mathrm{J}$, This estimate shows the promise of the approach. The estimate is valid on the time scale when the collisions in the plasma destroy the coherence. It occurs at times of order $\delta t=1 / \sigma c N \simeq 1 \mathrm{ps}$, where $\sigma$ is the atomic cross section for atomic collisions that destroy the excited coherence.

\section{CONCLUSION}

In this paper we have found several analytical solutions for a two-level atomic system under the action of a far-offresonant strong pulse of laser radiation. The solutions are given in terms of the Heun function, which is a generalization of the hypergeometric function. The Rosen-Zener and BambiniBerman models belong to this class of pulses as special cases. A better approximation for the box pulse is also obtained here, which takes care of nonanalyticity at the edges by introducing a parameter $\delta$. The results obtained here have applications to the generation of XUV radiation and the estimate reported here shows good potential as a source of coherent radiation. The technique used here to get the exactly solvable pulse shapes can be generalized to appropriate time-dependent detuning $\Delta=\Delta(t)$ cases and produce more exactly solvable models (to be reported elsewhere) [48].

\section{ACKNOWLEDGMENTS}

We thank M. O. Scully, L. Keldysh, M. S. Zubairy, V. A. Sautenkov, H. Eleuch, A. Svidzinsky, H. Li, and E. Sete for useful discussions. We also gratefully acknowledge the support from NSF Grant No. EEC-0540832 (MIRTHE ERC), the Defense Advanced Research Projects, Office of Naval Research (Grant Nos. N00014-09-1-0888 and N00014081-0948), and the Robert A. Welch Foundation (Award No. A-1261) and partial support from the CRDF. P.K.J. would also like to acknowledge the Robert A. Welch Foundation for financial support.

\section{APPENDIX: GENERATION OF RADIATION BY A TWO-LEVEL ATOMIC MEDIUM WITH EXCITED COHERENCE}

Let us assume that a TLA has some small initial coherence $\rho_{a b}^{0}=\sqrt{\rho_{a a}^{0} \rho_{b b}^{0}}$. Note that in this paper, we consider the case where there is no population inversion, $\rho_{a a}^{0}<\rho_{b b}^{0}$. The density matrix equations for atomic coherence are

$$
\begin{gathered}
\frac{\partial \rho_{a b}}{\partial t}=i \Omega\left(\rho_{a a}-\rho_{b b}\right), \\
\frac{\partial}{\partial t}\left(\rho_{a a}-\rho_{b b}\right)=-2 i \Omega \rho_{a b} ;
\end{gathered}
$$

the solution (by neglecting relaxation processes) is

$$
\rho_{a b}=i \rho_{a b}^{0} \sin \theta \text {. }
$$

Then, for the retarded frame,

$$
\tau=t-\frac{z}{c}
$$

the propagation equation for a resonant field is given by

$$
\frac{\partial \Omega}{\partial z}=-i \eta \rho_{a b},
$$

where $\eta=3 \lambda^{2} N \gamma /(8 \pi)$ is the coupling constant. Introducing

$$
\theta=2 \int^{t} \Omega d t,
$$

Equation (A5) can be rewritten as

$$
\frac{\partial^{2} \theta}{\partial z \partial \tau}=-\eta \sin (\theta-\phi),
$$

where $\phi$ can be determined from initial condition as

$$
\phi \simeq 2 \sqrt{\rho_{a a}^{0}} .
$$


Solution of Eq. (A7) is given by

$$
\theta=\phi\left[1-J_{0}(2 \sqrt{\eta z \tau})\right],
$$

and the Rabi frequency is

$$
\Omega=\phi J_{1}(2 \sqrt{\eta z \tau}) \sqrt{\frac{\eta z}{\tau}} .
$$

The energy of the generated short-wavelength pulse can be calculated as

$$
\frac{c}{4 \pi} A \int_{-\infty}^{\infty}|E|^{2} d t=A z N \rho_{a a} \hbar \omega_{a b},
$$

and it is equal to the energy stored in the medium after excitation. Also, it is important to note that the absence of population inversion does not influence the pulse energy much because of coherent interaction of the radiation field with the atomic medium.

The time duration of the generated pulse is of the order of

$$
\tau_{\text {pulse }}=\frac{4 \pi}{3 N \lambda^{2} z \gamma_{r}},
$$

and it gives the power of the pulse by

$$
P_{\text {pulse }}=\left[\frac{\lambda^{2} z N}{4 \pi}\right] A z N \gamma_{r} \rho_{a a} \hbar \omega_{a b},
$$

where the factor $\lambda^{2} z N / 4 \pi$ shows the brightness of the source in comparison with spontaneous emission of the incoherent source.
[1] M. O. Scully and M. S. Zubairy, Quantum Optics (Cambridge University Press, Cambridge, UK, 1997).

[2] L. Allen and J. H. Eberly, Optical Resonance and TwoLevel Atoms (Cambridge University Press, Cambridge, UK, 1997).

[3] B. W. Shore, The Theory of Coherent Atomic Excitation (Wiley, New York, 1990).

[4] K.-A. Suominen, B. M. Garraway, and S. Stenholm, Opt. Commun. 82, 260 (1991).

[5] K.-A. Suominen and B. M. Garraway, Phys. Rev. A 45, 374 (1992).

[6] S. Stenholm, Laser Phys. 15, 1421 (2005).

[7] N. Rosen and C. Zener, Phys. Rev. 40, 502 (1932).

[8] F. Bloch and A. Siegert, Phys. Rev. 57, 522 (1940).

[9] M. A. Nielsen and I. L. Chuang, Quantum Computation and Quantum Information (Cambridge University Press, Cambridge, UK, 1990).

[10] H. C. Torrey, Phys. Rev. 76, 1059 (1949).

[11] H. Salwen, Phys. Rev. 99, 1274 (1955).

[12] G. M. Genkin, Phys. Rev. A 58, 758 (1998).

[13] A. Plucinska and R. Parzynski, J. Mod. Opt. 54, 745 (2007).

[14] N. Rosen and C. Zener, Phys. Rev. 40, 502 (1932).

[15] M. V. Fedorov, Opt. Commun. 12, 205 (1974).

[16] M. V. Fedorov, Sov. J. Quantum Electron. 5, 816 (1975).

[17] I. I. Rabi, Phys. Rev. 51, 652 (1937).

[18] L. D. Landau, Phys. Z. Sowjetunion 2, 46 (1932).

[19] Y. N. Demkov, Sov. Phys. JETP 18, 138 (1964).

[20] F. T. Hioe, Phys. Rev. A 30, 2100 (1984).

[21] A. Bambini and P. R. Berman, Phys. Rev. A 23, 2496 (1981).

[22] J. Zakrzewski, Phys. Rev. A 32, 3748 (1985).

[23] E. E. Nikitin, Opt. Spectrosc. 13, 431 (1) (1969).

[24] N. V. Vitanov, J. Phys. B 27, 1791 (1994).

[25] A. M. Dykhne, Sov. Phys. JETP 11, 411 (1960).

[26] J. P. Davis and P. Pechukas, J. Chem. Phys. 64, 3129 (1976).
[27] M. O. Scully, Y. Rostovtsev, A. Svidzinsky, and J.-T. Chang, J. Mod. Opt. 55, 3219 (2008).

[28] Y. Rostovtsev, H. Eleuch, A. Svidzinsky, H. Li, V. Sautenkov, and M. O. Scully, Phys. Rev. A 79, 063833 (2009).

[29] J. D. Jackson, Classical Electrodynamics (Wiley, New York, 1962).

[30] K. Heun, Math. Ann. 33, 161 (1889).

[31] A. Ronveaux, Heun's Differential Equations (Oxford University Press, Oxford, 1995).

[32] N. Gurappa and P. K. Panigrahi, J. Phys. A Math. Gen. 37, L605 (2004).

[33] N. Gurappa, P. K. Jha, and P. K. Panigrahi, SIGMA 3, 057 (2007).

[34] R. S. Maier, Math. Comp. 76, 811 (2007).

[35] P. Jaegle, Coherent Sources of XUV Radiation (Springer, New York, 2005).

[36] P. Gibbon, Short Pulse Laser Interactions with Matter: An Introduction (Imperial College Press, London, 2005).

[37] Y. Rostovtsev and M. O. Scully, J. Mod. Opt. 54, 2607 (2007).

[38] Y. Rostovtsev, J. Mod. Opt. 55, 3149 (2008).

[39] Y. Rostovtsev, J. Mod. Opt. 56, 1949 (2009).

[40] K. H. Hahn, D. A. King, and S. E. Harris, Phys. Rev. Lett. 65, 2777 (1990).

[41] K. Hakuta, L. Marmet, and B. P. Stoicheff, Phys. Rev. Lett. 66, 596 (1991).

[42] G. Z. Zhang, K. Hakuta, and B. P. Stoicheff, Phys. Rev. Lett. 71, 3099 (1993).

[43] Y. Li and M. Xiao, Opt. Lett. 21, 1064 (1996).

[44] M. Jain, H. Xia, G. Y. Yin, A. J. Merriam, and S. E. Harris, Phys. Rev. Lett. 77, 4326 (1996).

[45] R. W. Boyd and M. O. Scully, Appl. Phys. Lett. 77, 3559 (2000).

[46] N. G. Kalugin and Y. V. Rostovtsev, Opt. Lett. 31, 969 (2006).

[47] V. A. Sautenkov, C. Y. Ye, Y. V. Rostovtsev, G. W. Welch, and M. O. Scully, Phys. Rev. A 70, 033406 (2004).

[48] P. K. Jha and Y. Rostovtsev (in preparation). 